\title{
Palate Obturators in the Phono-Articular System in Defects Caused by Nasal T / NK Cell Lymphoma
}

\author{
Lucia Guadalupe Robledo Carrizales, ${ }^{1 *}$ José Federico Torres Terán, ${ }^{2}$ Vicente Ernesto González Cardín ${ }^{2}$ \\ ${ }^{1}$ Graduated from the specialty of Maxillofacial Prosthesis FO UNAM, Mexico \\ ${ }^{2}$ Professor of the Maxillofacial Prosthesis Specialty at UNAM, Mexico
}

\begin{abstract}
Lymphomas are frequent malignant neoplasms, due to the destructive and rapidity of their clinical course, they present extensive areas of necrosis, which could be induced by the Epstein Bar virus (EBV) that is present in most of these lymphomas causing oro-nasal communications that they have an impact on phonation, chewing, swallowing and loss of self-esteem. Due to the extension of this type of communication, they represent a challenge in surgical rehabilitation opting for a prosthetic device.
\end{abstract}

\section{Introduction}

Lymphomas are a group of neoplasms originating in the immune system, whose cells are widely distributed and have an extensive functional range. In the head and neck region there is a high incidence of nasal type T / NK cell lymphomas associated with the Epstein Var virus (EBV) showing a similar pattern of strain type distribution between neoplastic tissues and reactive processes. EBV may infect cells of the T / NK lineage, raising the possibility of exposure of the nasal, nasopharyngeal, hard and soft palate mucosa. It is also argued that EBV may participate in the induction of cytokines, in particular tumor necrosis, factor kappa- $\beta$ and metalloproteases in virally infected cells, causing areas of necrosis. ${ }^{1}$ The oral cavity, the nasal cavity, the maxillary sinuses and the rest of the oropharynx area is constituted by the presence of the hard palate that structurally forms a physical barrier and the soft palate that together with a series of movements of the pharyngeal walls achieves the separation and coupling of the nasal space with the oral and pharyngeal space. The anatomical integrity of both is important to ensure proper function. Maxillary defects can be restored by means of a prosthetic device designed to close these acquired communications. A palatal obturator is defined as a prosthetic device designed to close a congenital or acquired communication by separating the oral cavity from the nasal or antral; whose main function should be to preserve teeth and remaining tissues in good condition. ${ }^{2}$ To produce speech, coordination between the respiratory system, the phonatory system, resonators and articulators is needed, that is, an air generator, a sound source, a resonator tract and an emitter system are needed. When there is a change in the maxillary morphology and the nasal geometry and a communication between the nasal cavity and the oral cavity, a change in nasal air flow and respiratory function is caused.

Components of respiratory function such as airflow volume, airway resistance, and functional residual volume can be affected by maxillary resection, leading to obvious reductions in air filtration, heating, and humidification causing accumulation of secretions in the defect area. ${ }^{3}$ The phono-respiratory system constitutes a functional unit that includes the control centers of the CNS, the lungs, the rib cage and the abdominal cavity, which work in an organized, precise and sequenced manner where a failure in one of its parts will be reflected on the others, the main function of these components is not phonation, however in man these organs are capable of
Quick Response Code:

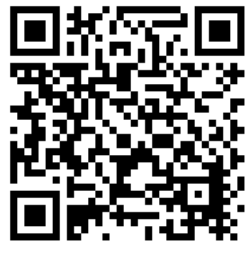

\footnotetext{
*Corresponding author: Lucía Guadalupe Robledo Carrizales, Graduated from the specialty of Maxillofacial Prosthesis FO UNAM, Melchor Ocampo 1327-A, Mexico, San Luis Potosí, SLP, Mexico

Received: 20 March, 2021

Published: 22 April, 2021

Citation: Lucia GRC, José FTT, Vicente EGC. Palate Obturators in the Phono-Articular System in Defects Caused by Nasal T / NK Cell Lymphoma. SOJ Complement Emerg Med. 2021;1(1):1-3. DOI: 10.53902/SOJCEM.2021.01.000504
} 
organizing themselves to emit sounds. During phonation there is a phenomenon of transduction of the aerodynamic energy generated by the respiratory system into acoustic energy radiated at the level of the lips, which is heard as sound. Pulmonary expiratory air is fragmented at the laryngeal level since this is a vibrating element and frequency regulator, which gives rise to harmonics from the vibration of the vocal cords, through effector air flow and accumulated pressure blows, this sound it will acquire its breadth and quality along the resonator tract constituted by the pharynx (hypopharynx, oropharynx and rhino pharynx), the nasal cavity and the oral cavity, phonemes, syllables \& words, the precise position of the articulators will determine the sound that the voice makes. ${ }^{4}$

A correct respiratory pattern is important to achieve adequate speech-respiratory coordination and, therefore, a good voice emission. The sound must begin at the same moment in which the expiration begins and the vocal cords approach beginning the vibratory cycle, since otherwise an uncoordinated start will be made and a strong or blown glottis blow may be heard. One of the main impacts of oro-nasal communication is the deterioration of speech understanding. Communication between the oral and nasal cavities reduces intraoral air pressure during speech production causing articulatory imprecision, hyper nasal speech, nasal air emission, and reduction in vocal volume that causes impaired speech intelligibility, which interferes with quality of life. ${ }^{5}$

\section{Methodology}

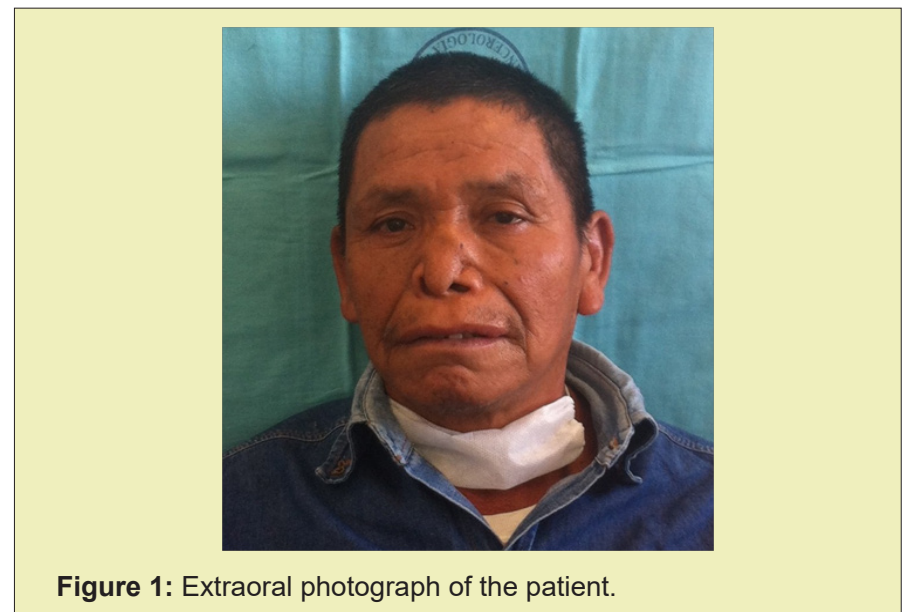

Figure 1: Extraoral photograph of the patient.

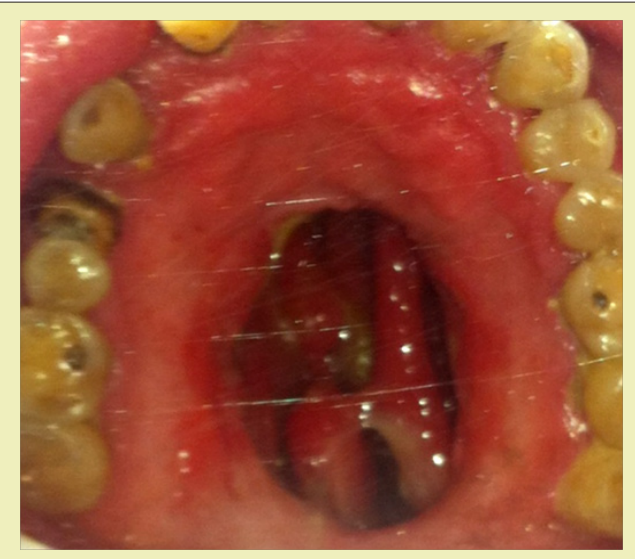

Figure 2: Image of oral and nasal communication.
A 55-year-old male patient, diagnosed with nasal T / NK cell lymphoma, in the clinical evaluation a perforation in the upper maxilla is observed at the medial and central level, which produces nasality to phonation with a decrease in the range of understanding of the same. The dental status is verified, and an occlusal adjustment is made in order to achieve the placement of metal hooks that will hold the obturator. An impression is taken and the design and processing of the shutter is carried out Figure 1-3.

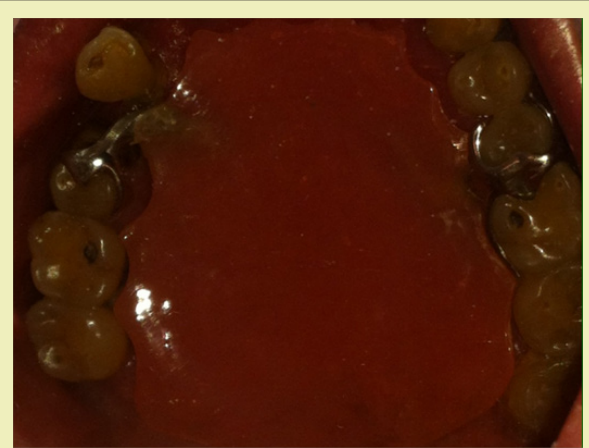

Figure 3: Plug in and in position.

\section{Results}

The components of phonation that are directly influenced by the shutter are respiration, resonance, and articulation. In both cases, an attempt should be made to achieve a uniform subglottic pressure that reduces turbulence and noise in addition to prolonging phonation times, paying special attention to the control of oral resonance and taking into account the elasticity of the orofacial and velopharyngeal muscles as well as taking advantage of the mobility of the pharyngeal walls that they will try to compensate as long as the obturator allows it the deficit created in the velopharyngeal sphincter of the bulb so that these walls maintain their mobility, thus achieving a compensatory valve closure.

\section{Discussion}

When presenting an oro-nasal communication speech tends to become hypernasal and is often unintelligible, nasality and speech intelligibility measurements can be used to evaluate the efficacy of oronasal. ${ }^{6,7}$ Although the acoustic characteristics associated with nasality are not completely eliminated, they are significantly reduced with the prosthesis. Speech resonance results have shown a reduction in hypernasality with the use of the prosthesis in most patients (69.6\%), according to some studies in the literature, however, the success of prosthetic treatment may be limited by factors such as radiotherapy, the extent of the defect, and speech therapy. ${ }^{8}$ When evaluating the degree of nasality in speech, one must be cautious, mainly for two reasons: coarticulation (vowel sounds in normal speech can be accompanied by a certain degree of nasal resonance, which is usually unnoticed by the listener) and relative value of nasality in speech, since this nasality varies with languages and even within the same language with its regional variations. ${ }^{9}$

\section{Conclusion}

There are various causes for the existence of an oro-nasal communication, it is important the work of a multidisciplinary team 
that takes the best option to achieve a good, non-invasive rehabilitation since many of them will have to go through long cycles of chemotherapy to eradicate the lymphoma, it is better to opt for a prosthetic device and not attempt any surgical closure and prioritize the improvement in the patient's quality of life by returning functions such as speech, swallowing and the reduction of the psychological impact that this type of defects can cause in these patients, These procedures must always be performed by specialists in maxillofacial prosthetic rehabilitation and one of the most important conclusions is that with a relatively simple procedure we can make a big difference to the quality of life of the patient.

\section{Acknowledgments}

None.

\section{Funding}

None.

\section{Declaration of Interest}

Author declares that there is no conflict of interest.

\section{References}

1. Herrera GA, Granados GM. Manual of Oncology Surgical Medical Procedures. $5^{\text {th }}$ Edn. 923-939.
2. Beumer JIII MM. Maxillofacial Rehabilitation; Surgical and Prosthodontic Management of Cancer-Related. Acquired and Congenital Defect of the Head and Neck, Quintessence. 2011;155-212.

3. Nazareth CE, Faria J, Mion O, et al. Nasal Valve anatomy and physiology. Braz J Otothinolaryngol. 2009;75(2):305-310.

4. Dong X, Zhy C, Qian Y, et al. The influence of obturators on Respiration of Patients with Maxillary Defects: A Clinical Study. PLos One. 2015;10(5):e127597.

5. Gonzales AL, Sprekelsen C, Capitan A. Video chemography as part of the multitest study in the treatment of functional dysphonia. 2012.

6. Qian Y, Qian H, Wu Y, et al. Numeric Simulation of upper airway structure and airflow dynamic characteristics after unilateral complete maxillary resection. The international journal of prosthodontics. 2012;26(3):268271.

7. Yoshida H, Furuya Y, Shimodatra K, et al. Spectral characteristics of hypernasality in maxillectomy patients. Journal of oral Rehabilitation. 2000;27:723-730.

8. Carvalho V, Pegoraro MI, Pereira JR. Speech evaluation with and without palatal obturator patients submitted to maxillectomy. J Appl Oral. 2006;14(6):421-426.

9. Fernández R, García F. Velopharyngeal insufficiency associated with dysphonia and alteration of resonance. 2002. 